\title{
Construction And Analysis Of The Time-Evolving Pain-Related Brain Network Using Literature Mining
}

This article was published in the following Dove Press journal:

Journal of Pain Research

\section{Jihong Oh \\ Hyojin Bae (iD \\ Chang-Eop Kim (D)}

Department of Physiology, College of Korean Medicine, Gachon University,

Seongnam 13/20, Republic of Korea
Correspondence: Chang-Eop Kim Department of Physiology, College of Korean Medicine, Gachon University, I342, Seongnam-daero, Sujeong-gu, Seongnam-si, Gyeonggi-do, Repubic of Korea Tel/fax +82-3I-750-54I6

Email eopchang@gachon.ac.kr
Purpose: We aimed to quantitatively investigate how the neuroscience field developed over time in terms of its concept on how pain is represented in the brain and compare the research trends of pain with those of mental disorders through literature mining of accumulated published articles.

Methods: The abstracts and publication years of 137,525 pain-related articles were retrieved from the PubMed database. We defined 22 pain-related brain regions that appeared more than 100 times in the retrieved abstracts. Time-evolving networks of pain-related brain regions were constructed using the co-occurrence frequency. The state-space model was implemented to capture the trend patterns of the pain-related brain regions and the patterns were compared with those of mental disorders.

Results: The number of pain-related abstracts including brain areas steadily increased; however, the relative frequency of each brain region showed different patterns. According to the chronological patterns of relative frequencies, pain-related brain regions were clustered into three groups: rising, falling, and consistent. The network of pain-related brain regions extended over time from localized regions (mainly including brain stem and diencephalon) to wider cortical/ subcortical regions. In the state-space model, the relative frequency trajectory of pain-related brain regions gradually became closer to that of mental disorder-related brain regions.

Conclusion: Temporal changes of pain-related brain regions in the abstracts indicate that emotional/cognitive aspects of pain have been gradually emphasized. The networks of painrelated brain regions imply perspective changes on pain from the simple percept to the multidimensional experience. Based on the notable occurrence patterns of the cerebellum and motor cortex, we suggest that motor-related areas will be actively explored in pain studies.

Keywords: pain, pain-related brain regions, pain-related brain networks, pain research trend analysis, literature mining, text mining, mental disorders and pain

\section{Introduction}

Over the decades, enormous basic and clinical study efforts have led to many advances in the understanding of pain mechanism, and researchers have expanded their knowledge on the complex and multidimensional characteristics of pain. ${ }^{1-3}$ In the early investigation of the brain mechanism of pain, efforts have been made to find a single brain area responsible for pain perception, as in the other sensory modality of vision or hearing. However, it turned out that pain is multidimensional experience emerging from the integrated activity of the brain and there is no single region such as "primary pain cortex".

Numerous neuroimaging studies have demonstrated that multiple brain regions are involved in various pain conditions. While several brain regions such as the 
thalamus, insula, and ACC have been consistently reported to be activated during acute nociception regardless of the type of noxious stimuli, brain activity patterns for chronic pain are rather heterogeneous within and across different chronic pain conditions. However, studies from both acute and chronic pain have highlighted the emotional and cognitive aspects in pain perception regardless of the pain types. $^{4-10}$ Furthermore, accumulated evidence has indicated interactions between mental disorders and acute/ chronic pain. ${ }^{11-15}$ Recently, a new perspective was suggested which states that pain perception is associated with the negative moods (eg, anxiety and depression) as a continuum of aversive behavioral learning. ${ }^{16}$

There are hundreds of thousands of accumulated articles about pain so far. Many researchers have reviewed the activity of diverse brain regions involved in various pain conditions to understand the brain mechanisms of pain perception. However, it is practically limited for the researchers to manually investigate a vast number of papers and draw quantitative results efficiently. It also might be possible to obtain biased results according to the researcher's background knowledge or research interests. Recently, the literature mining approach has been actively applied in various biomedical fields to efficiently extract scientific knowledge from the accumulated data. ${ }^{17-25}$ Literature mining converts unstructured textual information into structured data to extract meaningful numeric information and find patterns. ${ }^{26,27}$ The advantage of literature mining is that it can quickly analyze vast quantities of documents and mine the latent knowledge such as the implicit relationships between the words by computing quantitative metrics, eg, the frequency of occurrence and co-occurrence between words.

In this study, we aimed to quantitatively investigate how the neuroscience field developed over time in terms of its concept on how pain is represented in the brain and compare the research trends of pain with those of mental disorders through literature mining of accumulated published articles. First, the bibliographic information of 137,525 pain-relevant abstracts was retrieved from PubMed and then preprocessed. The brain regions were automatically recognized from the abstracts. Subsequently, we performed frequency and cooccurrence analyses to identify the temporal pattern of the occurrences of pain-related brain regions. Relative frequency patterns of pain-related brain regions were compared with those of mental disorders-related brain regions. Evolving occurrence patterns of the pain-related brain regions were investigated through the network analyses and the statespace model (Figure 1). Furthermore, future trends in the pain study were suggested based on the evolving patterns of the pain-related brain network.

\section{Materials And Methods}

\section{Datasets}

\section{Bibliographic Information Retrieval}

We downloaded the abstracts and publication year information of articles from PubMed (https://www.ncbi.nlm.nih.gov/ pubmed/) using custom Python scripts and the Biopython Entrez module (http://biopython.org/DIST/docs/api/Bio. Entrez-module.html). "Pain[majr]" was used as the query to search articles whose MeSH Major Topics were classified as pain in PubMed. Only the abstracts published until 2015 were retrieved because the MeSH Major Topics of the papers published after 2016 were not fully classified in PubMed at the time of analysis (on May 14th, 2018). We also retrieved bibliographic information of the articles related to the representative mental disorders: schizophrenia, depression, anxiety disorders, bipolar disorder, and post-traumatic stress disorders.

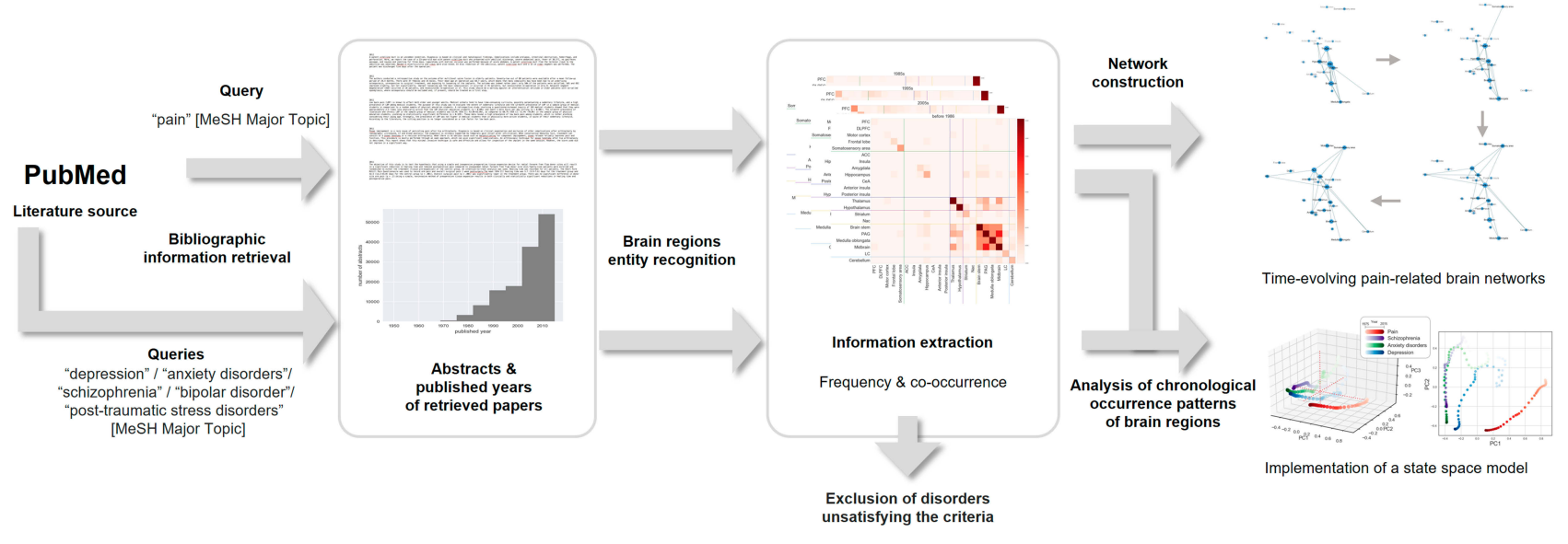

Figure I Overview of construction and analysis of the evolving patterns of the pain-related brain network using literature mining. 


\section{Compiling A Dictionary Of The Brain Regions}

It is a challenge to recognize the nomenclature of brain regions from the unstructured texts because they are nonstandardized. To compile a dictionary of the brain regions, we downloaded the ontology of brain regions from the Brede database (http://neuro.imm.dtu.dk/wiki/Brede Database/ WOROI), which includes 586 macroscopic brain regions in humans. To consider synonyms, we used the Neuroscience Information Framework Standard Ontology (NIFSTD; https://bioportal.bioontology.org/ontologies/NIFSTD).

\section{Recognition Of The Brain Regions In The Abstracts}

We used Python's regular expression module to preprocess the abstracts and automatically recognize brain regions of the compiled dictionary in the abstracts using a rule-based approach. The rule was used to recognize the brain region term with spaces before and after it in the abstract. With this rule, the occurrences of brain regions were computed in each abstract, and the occurrence matrix was constructed (Figure 2). First, the queries consisted of the preferred label (the primary term of the brain region in NIFSTD) and its synonyms. The binary occurrence matrix was constructed by recognizing all queries from the whole retrieved abstracts. As shown in Figure 2, each row represents the abstract; each column represents the brain region; and the element represents whether the given queries appear in the given abstract (value 1) or not (value 0 ).

\section{Determination Of The Pain-Related Brain Regions}

To determine the pain-related brain regions, we computed the occurrence frequencies of the regions by summing columns in the occurrence matrix. We selected the brain regions that appeared more than 100 times as pain-related brain regions. Then, a curation process was followed to sort out duplicated terms and remove the terms that are too broad (eg, brain, cerebral cortex, and white matter) or non-localizable (eg, cerebrospinal fluid).

\section{Analysis Of The Relative Frequency Of Pain-Related Brain Regions}

To identify significant patterns of pain-related brain regions in each year, we computed the relative frequencies of the pain-related brain regions as occurrence frequency divided by the number of abstracts including pain-related brain regions in the corresponding year.

In order to cluster the brain regions according to the changing patterns of the relative frequency over time, the first derivatives of the relative frequency were calculated for brain regions and clustered into three groups by applying $\mathrm{K}$-means clustering.

\section{Co-Occurrence Matrix Construction}

\section{And Network Analysis}

In literature mining, words are considered correlated when they are collocated in a corpus. To investigate the change of region-to-region associations, we conducted co-occurrence analysis to quantify co-occurrences of the brain regions in the abstracts.

First, the temporal patterns of co-occurrences between brain groups were identified. Pain-related brain regions were assigned to the following six groups: cortex region (CTX), limbic area (Limbic), diencephalon (DIEN), basal ganglia (BG), brain stem (BS), and cerebellum ( $\mathrm{Cb}$ ). The group cooccurrence frequencies were calculated in every year of

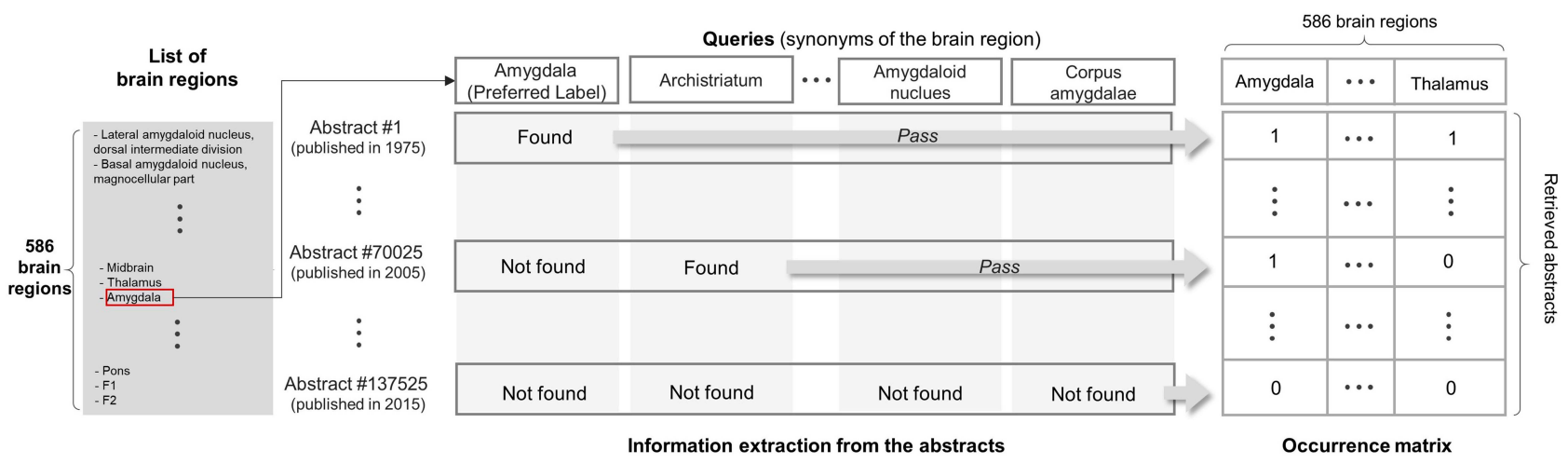

Figure 2 Workflow of recognition of brain regions in the abstracts and construction of the occurrence matrix. Brain regions from the Brede database were listed. The query for the recognition consisted of synonyms of each brain region. The occurrence matrix was constructed by recognizing all queries in the abstracts. Each row represents the abstract; each column represents the brain region; and the element represents whether the given queries appear in the given abstract (value I) or not (value 0). 
publication and were divided by the number of pairs constituting the group, thereby minimizing the bias due to the different number of regions between the groups. Considering that the total number of studies increases over the years, we normalized the group co-occurrence frequencies again to the total co-occurrence frequencies of each year.

We generated the annual co-occurrence matrices; their rows and columns indicate the pain-related brain regions, and each element contains the number of abstracts in which a pair of brain regions co-occur. To investigate the overall co-occurrence patterns of pain-related brain regions, we grouped annual co-occurrence matrices into the following four stages: stage 1 (before 1986), stage 2 (1986-1995), stage 3 (1996-2005), and stage 4 (20062015). Then, the elements of four co-occurrence matrices were divided by the total co-occurrence frequencies of the corresponding stage to identify the relatively important relationship of pain-related brain regions at each stage.

Subsequently, pain-related brain networks were constructed to investigate the topology of the interconnected brain regions at each stage. Each network consisted of nodes (pain-related brain regions) and edges between them (co-occurrences). For the tractability of analysis, the edges of the networks were binarized (1, connected; 0 , not connected) as follows. First, different co-occurrence thresholds were chosen in different networks to set the same edge density between networks. Second, we eliminated the edges with co-occurrences of less than 5 to avoid detecting spurious relationships. Networks were visualized and analyzed using Cytoscape 3.5.1 (http://www.cytoscape.org/). ${ }^{28}$

\section{Implementation Of The State-Space Model}

To compare the occurrence patterns of the brain regions in pain and mental disorders, the state-space model was implemented by applying the principal component analysis (PCA), a linear dimensionality reduction method. PCA finds a reduced set of new variables through a linear combination of initial variables while preserving the information as much as possible (ie, maximizing the total variance of the original data). ${ }^{29}$ In other words, these reduced set of variables called principal components (PCs) represent the direction that covers a maximal amount of variance in the high-dimensional space. The obtained low-dimensional representation of the data is composed of a much smaller set of variables and thus can be easily visualized.

For the pain- or mental disorders-related brain regions that appeared more than 100 times in the abstracts of each topic, we assigned the regions into ten groups: PFC, frontal cortex (Frontal), parietal cortex (Parietal), temporal cortex (Temporal), Limbic, DIEN, BG, BS, Cb, and corpus callosum (Corpus). A relative frequency matrix, consisting of 41 rows (year of publication) and 10 columns (brain region groups), was obtained from each disorder. The data were scaled into unit length vectors by dividing them by the norm of each column to focus on the vector direction rather than the magnitude. After concatenating the matrices, PCA was applied to project data resided in ten-dimensional space to three-dimensional space composed of PC1, PC2, and PC3. These projected data form the low-dimensional representation of each disorder which effectively summarizes the original data while minimizing the loss of information. In our study, these representations indicate the evolving trajectory of the relative frequency of the pain- and mental disorderrelated brain regions so that we could intuitively capture the trend of how the occurrence patterns of brain regions have changed over time. We plotted the data points to the two- and three-dimensional space after smoothing with the Gaussian kernel (sigma $=2$ ) to depict the trends effectively. Furthermore, we investigated the PCA coefficients of each brain region in given PCs to identify the contribution of each region in the moving direction of the trajectory.

\section{Results}

\section{Different Occurrence Patterns Of Brain Regions Over Time}

We identified the number of abstracts over time, along with the trends of the occurrence of the brain regions. A total of 137,525 pain-related abstracts published until 2015 were found in PubMed, and the number of abstracts substantially increased over time (Figure 3A). We constructed a dictionary of brain regions consisting of 586 brain regions from the Brede database and their synonyms obtained from NIFSTD. As shown in Figure 3B, the number of abstracts including brain regions also grew over time. However, different patterns were observed for the number of abstracts containing each brain region. For example, the number of abstracts containing PFC was few in the early years but increased from 1995, and the number of abstracts containing midbrain and striatum did not show steadily increasing patterns.

\section{Identifying Clusters Of Pain-Related Brain Regions According To The Changing Patterns Over Time}

We defined 22 pain-related brain regions as those appeared more than 100 times in the retrieved abstracts 


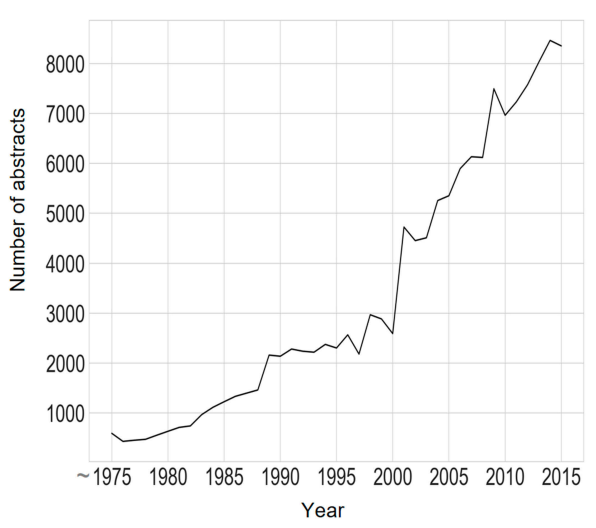

B

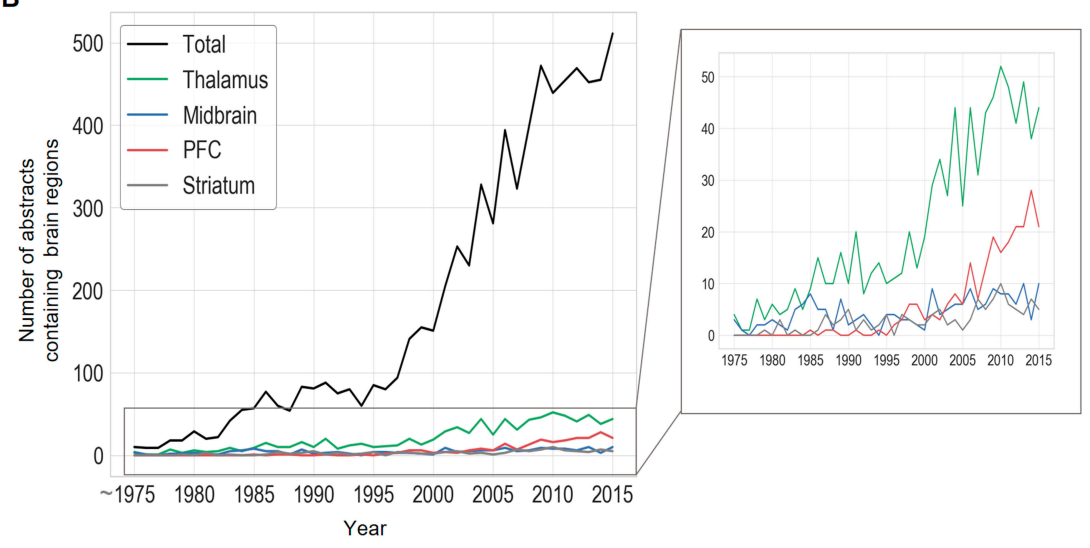

Figure 3 Changes in the number of pain-related abstracts over time. (A) The growth number of published pain-related abstracts retrieved from PubMed. (B) The total number of increasing abstracts containing 586 brain regions from the compiled dictionary (black line); examples of different patterns of the number of abstracts containing single brain region with different colors (green line, thalamus; blue line, midbrain; red line, PFC; gray line, Striatum).

Abbreviation: PFC, prefrontal cortex.

(Table 1). To investigate the chronological changes in the relative importance of pain-related brain regions, we calculated the first derivatives of the relative frequency for brain regions and clustered them into three groups by applying K-means clustering. As shown in Figure 4, the three clustered groups represent a pattern in which the occurrence frequency is generally increased, relatively consistent, or generally decreased with time. So, we named each group as rising, consistent, and falling. PFC, anterior insula, dorsolateral PFC (DLPFC), central amygdaloid nucleus (CeA), hippocampus, motor cortex, $\mathrm{Cb}$, Insula, ACC, somatosensory cortex, and amygdala were grouped into the rising cluster; midbrain, hypothalamus, periaqueductal grey $(\mathrm{PAG}), \mathrm{BS}$, and thalamus were grouped into the falling cluster; the rest were grouped into the consistent cluster.

\section{Change In Co-Occurrence Patterns Between Pain-Related Brain Regions}

We next attempted to figure out the changing pattern of the inter-regional relationship through the co-occurrence analysis because it has been demonstrated in recent years that pain is an integrated activity between multiple brain regions. Indeed, we found that the number of abstracts with more than two brain regions increased over time, while the number of abstracts with only one brain region decreased (Figure 5).

We quantified relative co-occurrence changes between the groups of pain-related brain regions and found that eight pairs of groups exhibited increasing or decreasing patterns (Figure 6). Pain-related brain regions were assigned to six groups as follows: (1) CTX group: PFC, DLPFC, motor cortex, frontal lobe, and somatosensory area; (2) Limbic group: ACC, insula, amygdala, hippocampus, CeA,

Table I Pain-Related Brain Regions And Their Occurrences In Pain-Related Abstracts

\begin{tabular}{|l|l|l|l|}
\hline Pain-Related Brain Regions & Occurrences & Pain-Related Brain Regions & Occurrences \\
\hline Thalamus & 849 & Prefrontal cortex (PFC) & 230 \\
Brain stem & 704 & Midbrain & 183 \\
Anterior cingulate cortex (ACC) & 673 & Cerebellum & 171 \\
Periaqueductal grey (PAG) & 611 & Anterior insula & 161 \\
Somatosensory area & 526 & Locus coeruleus (LC) & 152 \\
Insula & 493 & Nucleus accumbens (Nac) & 147 \\
Medulla oblongata & 475 & Frontal lobe & 128 \\
Amygdala & $38 I$ & Dorsolateral prefrontal cortex (DLPFC) & 125 \\
Motor cortex & 358 & Striatum & 116 \\
Hypothalamus & 328 & Central amygdaloid nucleus (CeA) & 105 \\
Hippocampus & 298 & Posterior insula & 103 \\
\hline
\end{tabular}


A

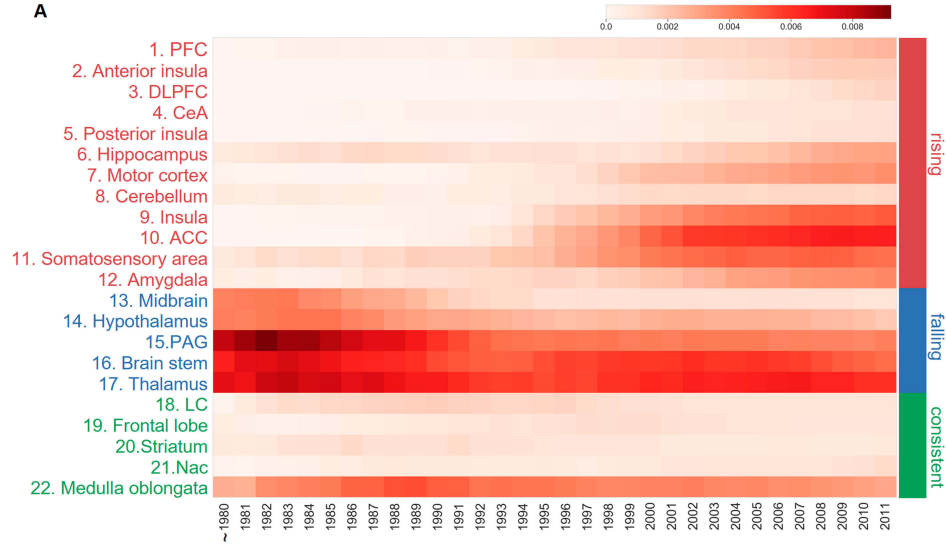

B

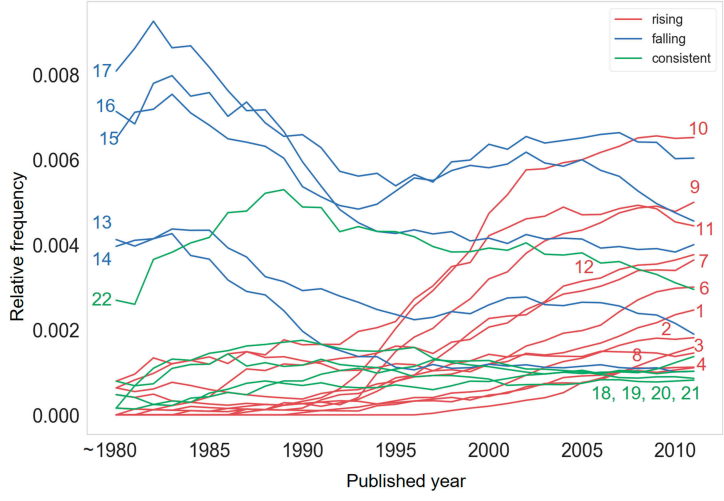

Figure 4 The relative frequency changes of pain-related brain regions over time. (A) Relative frequency changes of 22 pain-related brain regions over time were represented as a color matrix. Color bar (upper right) indicates the relative frequency values (the number of abstracts containing each brain region divided by the total number of abstracts in each year). Note that brain regions were clustered by K-means clustering according to the pattern of changes in relative frequencies (grouped into 3 clusters: rising, falling, and consistent). (B) Relative frequencies of pain-related brain regions were plotted against time in years. Each number label of the line corresponds to the label in the left matrix. A sliding window was used to smooth the values of matrix and graph (window size $=10$ ).
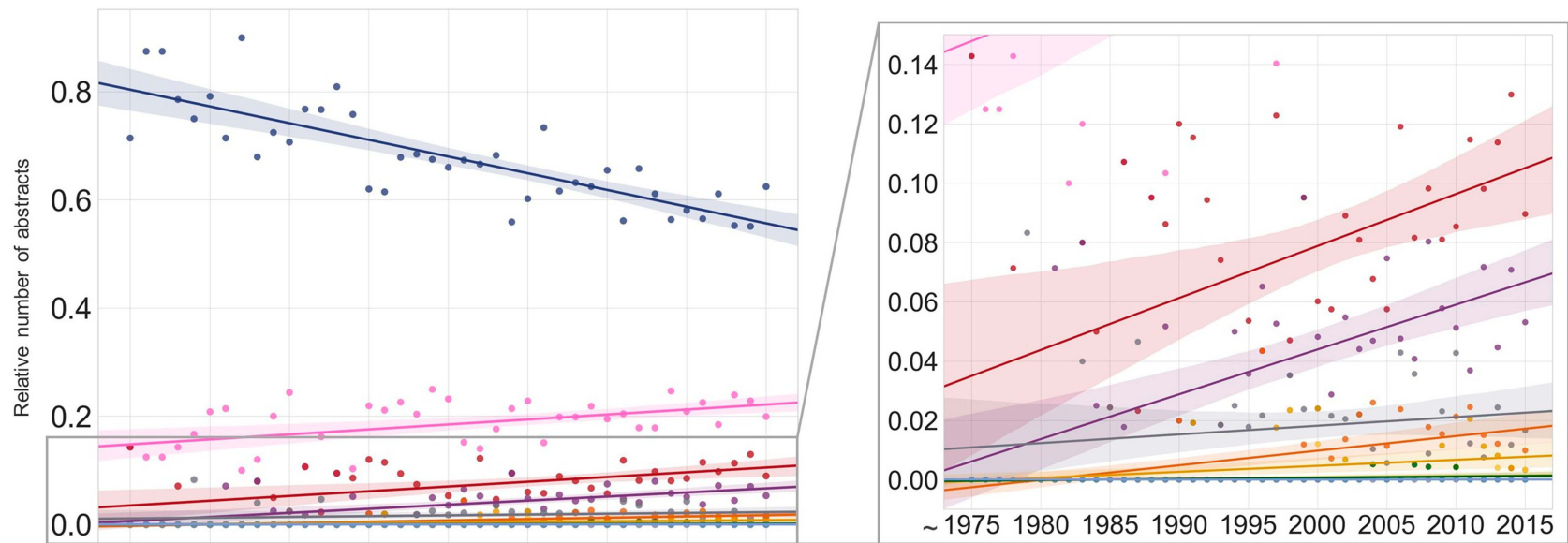

〜 197519801985199019952000200520102015

Published year

Number of pain-related brain regions

- $1 \bullet 2 \bullet 3$

4

5

6

$7 \bigcirc 8$

- $9 \bigcirc 10$

Figure 5 The relative number of pain-related abstracts containing different numbers of pain-related brain regions. Scatterplots with regression lines show that more pain-related brain regions co-occur in the abstracts with time. Each point with a different color represents the relative number of abstracts containing the indicated number of pain-related brain regions in the year. The number of abstracts was normalized by the number of those containing at least one brain region for computing relative number of abstracts.

anterior insula, and posterior insula; (3) DIEN group: thalamus and hypothalamus; 4) BG group: striatum and Nac; 5) BS group: brain stem, PAG, medulla oblongata, midbrain, and LC; 6) Cb group: cerebellum. Limbic and CTX tended to increasingly co-occur with other groups (DIEN-Limbic, Limbic-Limbic, DIEN-CTX, Limbic-CTX, and CTXCTX), while BS tended to co-occur less often with other groups (BS-BS and DIEN-BS) over time. Notably, although cerebellum showed low levels of relative frequency in general, it showed increasing patterns both in relative frequency and co-occurrence analysis (Figures 4 and 6).

\section{Construction Of Time-Evolving Pain- Related Brain Networks}

To investigate the topology of interconnected pain-related brain regions, we constructed the chronological weighted cooccurrence matrices and binarized pain-related brain networks over four stages (stage 1, before 1986; stage 2, 1986-1995; 


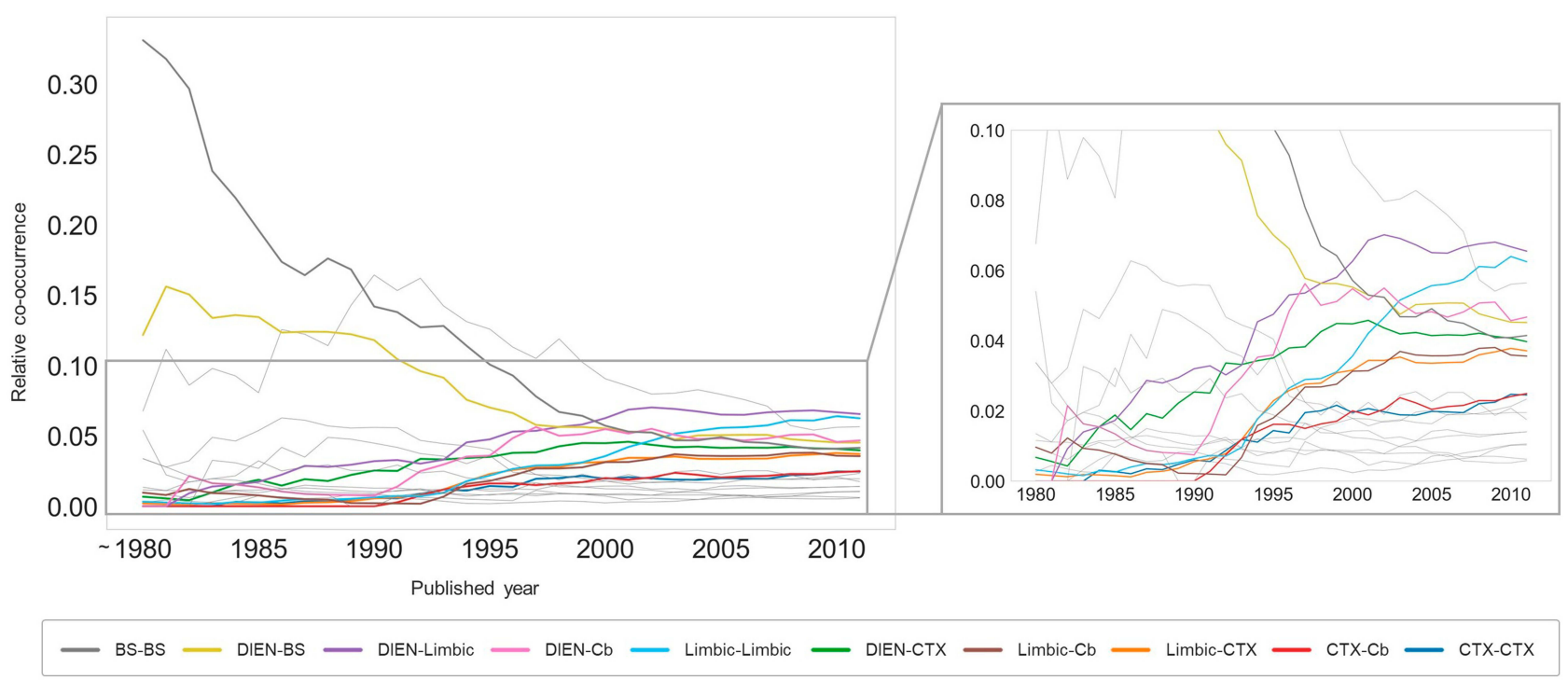

Figure 6 Relative co-occurrence changes of pain-related brain region groups over time. Relative co-occurrence changes were plotted against published years. The sliding window method was used to smooth the graph (window size $=10$ ).

Abbreviations: CTX, cortex region; DIEN, diencephalon; Limbic, limbic area; BG, basal ganglia; BS, brain stem; Cb, Cerebellum.

stage 3, 1996-2005; and stage 4, 2006-2015) (Figure 7). The binarized networks were constructed with an edge density of 0.09 for stages $2-4$. In stage 1 , the regions of BS group (medulla oblongata, PAG, and brain stem) and DIEN group (thalamus) co-occurred prevalently while other pain-related brain regions neither frequently occurred nor formed edges in the network. In stage 2, limbic areas such as the hippocampus and amygdala showed not only a significantly increased relative frequency but also an increased association with other regions including the hypothalamus, striatum, and PAG. Although the relative frequency was low, the somatosensory area was connected with the thalamus. In stage 3 , the network became extensive as new connections between ACC, insula, thalamus, $\mathrm{PFC}$, and somatosensory area emerged. The regions of the Limbic and CTX groups showed consistently high relative frequencies and tended to appear frequently with other brain regions in stages 3 and 4 . The brain stem still appeared at a high relative frequency, but the occurrences of other areas in the BS group (medulla oblongata, PAG, midbrain, and LC) in stage 4 decreased compared with those in other stages.

To evaluate important regions on the network topology, we analyzed the degree (the number of edges) of nodes in the networks. The subnetworks of hub regions (defined as the nodes with degrees $\geq 3$ ) and non-hub regions connected to the hubs were represented for every stage (Figure 8). PAG and thalamus had multiple edges in every stage. Midbrain, and medulla oblongata frequently co-occurred with other regions in stages 1 and 2, but not in stages 3 and 4 . Co- occurrences of the brain stem gradually decreased, and it was eventually excluded from the hub regions in stage 4 . On the other hand, ACC, insula, PFC, amygdala, and the somatosensory area that belonged to the rising cluster were found as the frequently co-occurred regions in stages 3 and 4 . Interestingly, we found that most of the regions of rising cluster (somatosensory area, ACC, insula, amygdala, motor cortex, hippocampus, and PFC) except for the motor cortex, posterior insula, and DLPFC formed edges with other brain regions in at least one subnetwork.

Hub nodes tended to form edges between themselves but were occasionally connected by edges with non-hub nodes. We attempted to seek potentially important regions in the subnetwork by focusing on non-hub nodes in terms of network topology. It was found that most non-hubs in a certain stage appeared as hubs in other stages, implying that the regions of the subnetworks are generally homogeneous. However, we found that the cerebellum that belonged to subnetworks as non-hubs in stage 3 and 4 had never been a hub in all stages. This suggests a possibility of the cerebellum as a potential hub in the future.

\section{Comparison Of Changing Patterns Of Brain Regions Between Pain And Mental Disorders In The State-Space}

We found that the importance of the brain areas concerning the emotional and cognitive aspects of pain has increased over time. The state-space model was then 

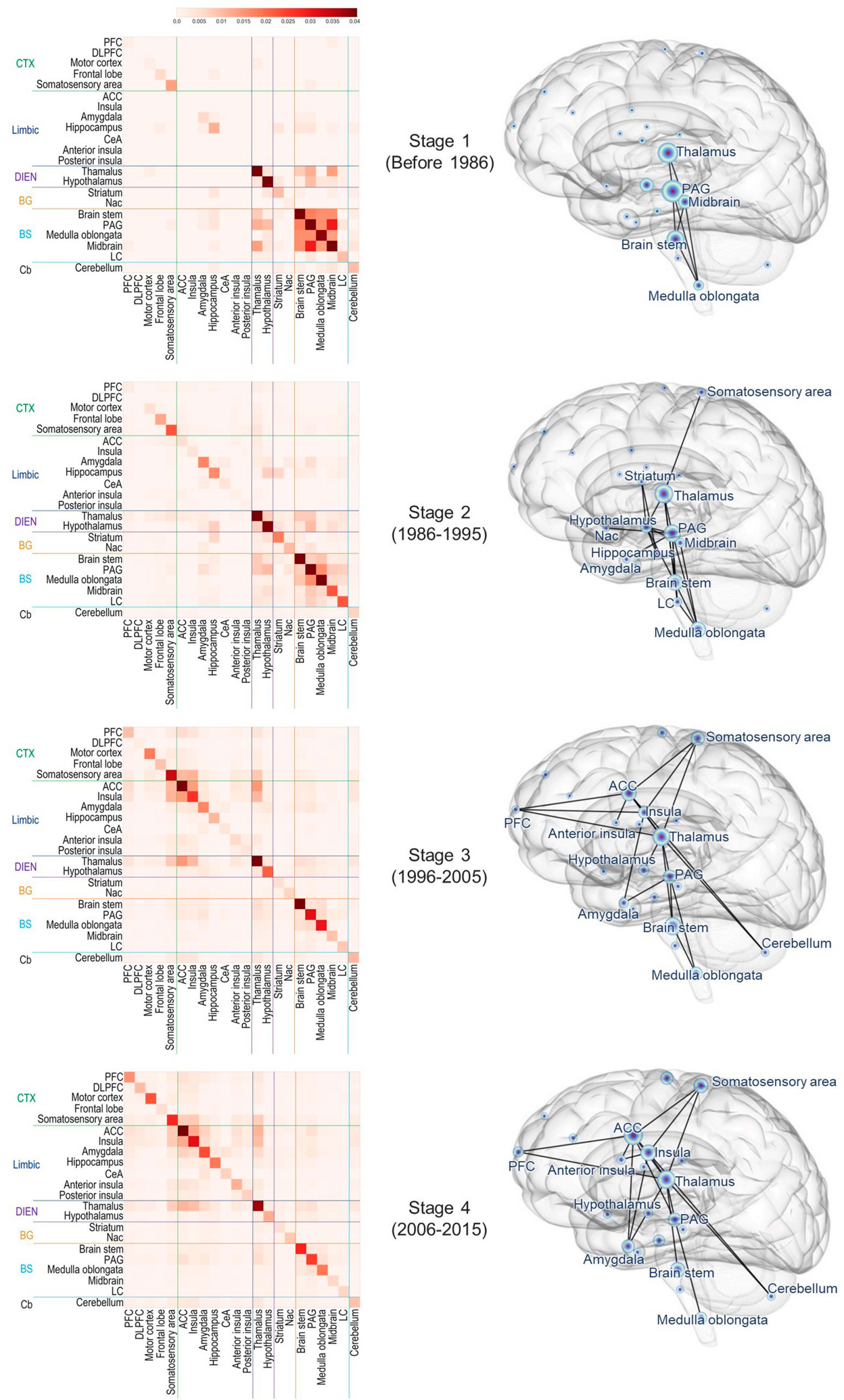

Figure 7 Chronological weighted co-occurrence matrices (left) and brain networks related to pain (right). Weighted networks were converted into binary networks by applying thresholds for tractability of analyses (varying thresholds to set the edge density constant across networks: edge density $=0.09$ ). In the visualized network (right), a node represents the pain-related brain region, and an edge between two nodes represents co-occurrence in abstracts. The node size is proportional to the relative frequency. 

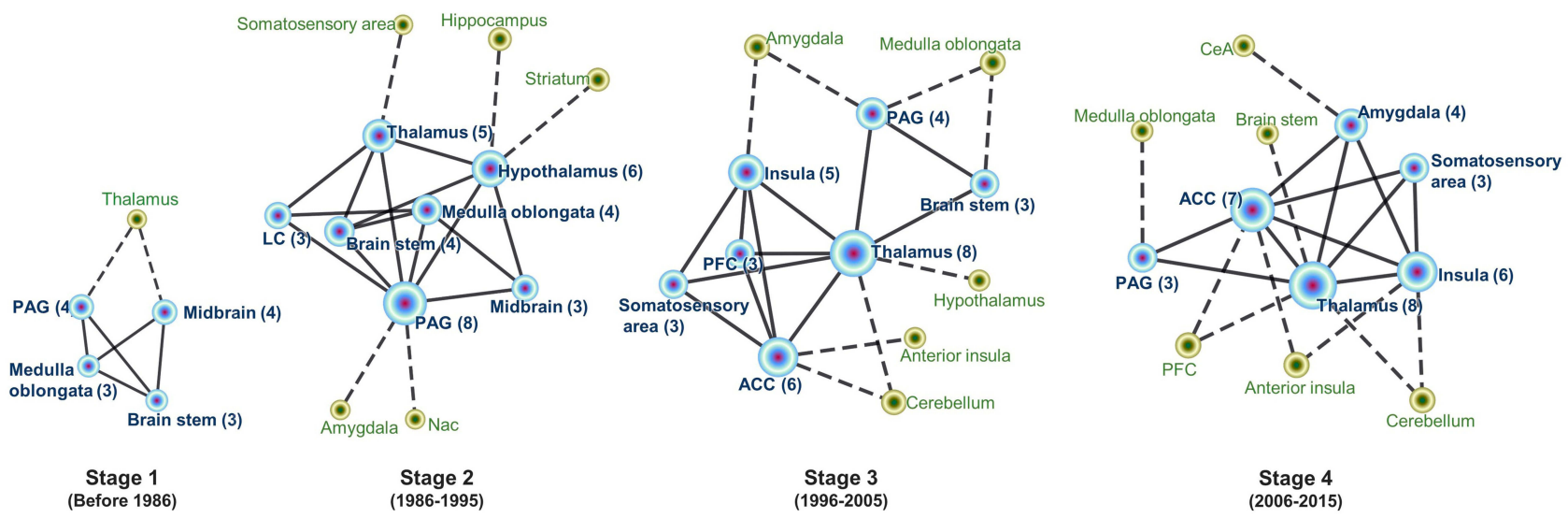

Figure 8 Subnetwork of hub brain regions (degree $\geq 3$ ) from the pain-related brain networks in each stage. Nodes with blue labels represent hub brain regions; nodes with green labels represent non-hub brain regions. The node size is proportional to the degree, and the degree of the hub nodes is indicated in parentheses.

implemented for more accurate analysis to compare the relative frequency patterns of brain regions in pain and mental disorders. By visualizing the trajectory of painrelated and mental disorder-related brain regions in the low-dimensional space, we could intuitively capture the occurrence patterns of the brain regions in pain and each mental disorder.

Of the mental disorders considered (schizophrenia, depression, anxiety disorders, bipolar disorder, and posttraumatic stress disorders), only those with at least 10 regions that appeared more than a hundred times by 2015 were included in the analysis: schizophrenia, anxiety disorder, and depression (Supplementary Table1). The tendimensional matrix composed of 41 years (row) and 10 brain region groups (columns) was reduced to a threedimensional matrix (41 by 3 ) by applying PCA for pain and mental disorders (See methods for detail). Reduced data representing the changing patterns of the relative frequency of brain regions in pain and mental disorders were plotted in the 2- and 3-dimensional space composed of $\mathrm{PC} 1$ and $\mathrm{PC} 2$, and $\mathrm{PC} 1, \mathrm{PC} 2$, and $\mathrm{PC} 3$, respectively (Figure 9). The three components (PC1, PC2, and PC3) explained $43 \%, 22 \%$, and $9 \%$ variances in the dataset respectively. The relative frequency trajectory of brain

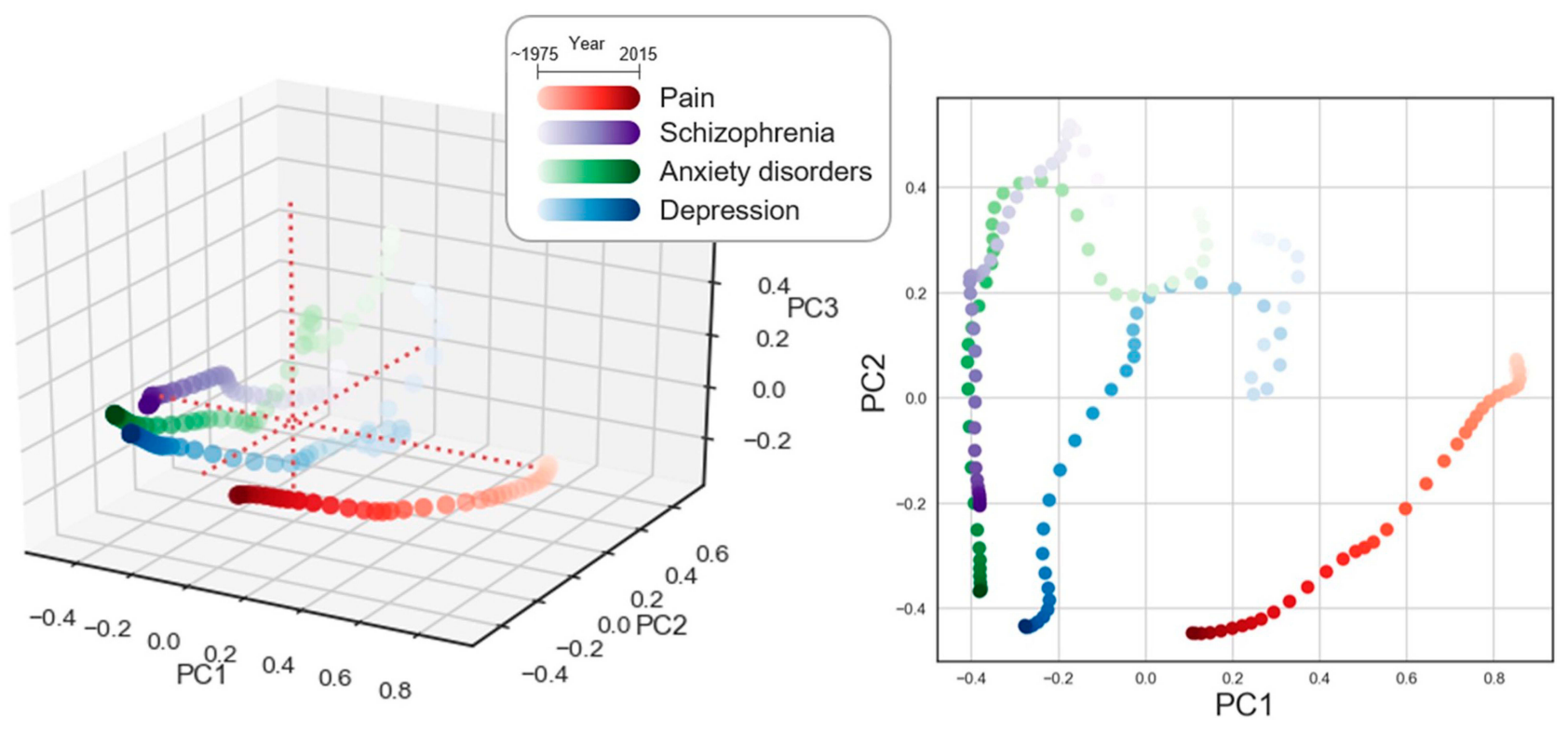

Figure 9 State-space model of brain regions related to pain and mental disorders (schizophrenia, anxiety disorders, and depression). A three-dimensional state-space model was implemented by applying PCA (left). The first two principal components were visualized in the two-dimensional space (right). The PC values of each disease were depicted as scatter plots of different colors. The gradient color changes represent the different publication years of the abstracts as described in the box. The scatter plots were smoothed using the Gaussian kernel $($ sigma $=2)$. 
regions related to pain shifted closer to that of brain regions related to mental disorders over time, suggesting that brain regions emerging in the abstracts related to the mental disorders and pain gradually became similar. The trajectory of pain-related brain regions, in particular, showed dramatic movement to a negative direction along the $\mathrm{PC} 1$ axis over time, thus approaching to the trajectory of mental disorder-related brain regions.

To interpret the movement of trajectory along the PC1 axis, we investigated the coefficients of PC1. Using PCA coefficients, we could quantitatively investigate the influence of each brain region group on constructing the trajectory (Table 2). For instance, BS and DIEN have large positive coefficients for $\mathrm{PC} 1$, which means these areas largely contribute to moving in a positive direction on the $\mathrm{PC} 1$ axis. Therefore, the trajectory of pain-related brain regions shifting to a negative direction in the $\mathrm{PC} 1$ axis implies that portions of BS and DIEN in the relative frequency of pain-related brain regions have decreased. In contrast, PFC, Limbic, and BG showed large negative coefficients for PC1, implying their increasing portions to the pain-related brain regions over time.

\section{Discussion}

In this study, we text-mined brain regions that were frequently mentioned in 137,525 pain-related abstracts in the PubMed database to quantitatively investigate how the neuroscientific field developed over time in terms of its concept on how pain is represented in the brain and compare the research trends of pain with those of mental disorders. We analyzed frequencies and co-occurrences of pain-related brain regions and compared the relative frequency patterns of pain-related brain regions with those of depression, anxiety disorders, and schizophrenia. We found that the brain regions in the pain-related abstracts have gradually extended, reflecting the changes of the perspective on pain from a simple modality of perception into a multidimensional experience. The relative frequency pattern of pain-related brain regions shifted closer to that of mental disorders-related brain regions in the state-space model.

The results of this study indicate that researchers have gradually come to focus on the emotional/cognitive aspects of pain rather than simple pain perception and modulation. We found brain regions that are highly related to mental disorders (such as PFC, amygdala, insula, ACC, and hippocampus) have shown increased relative frequencies and co-occurrences over time. $^{30}$ Similarly, there were common pain-related brain regions that appeared more than 100 times in the abstracts related to depression, anxiety disorders, and schizophrenia: PFC, DLPFC, frontal lobe, striatum, ACC, insula, hippocampus, and amygdala. These regions are known to be associated with cognitive and emotional processing, and most of them except frontal lobe and striatum belonged to the rising cluster on the relative frequency in our analyses. ${ }^{5,31,32}$ Recently, it has been suggested that pain perception is related to negative moods (eg, anxiety and depression) and is a continuum of aversive behavioral learning. ${ }^{16}$ The comorbidity of pain and mental disorders are common, and the correlation between pain and mental disorders has been widely investigated clinically. ${ }^{14,33-35}$ Especially, depression and anxiety disorders have been demonstrated to be highly correlated with chronic pain based on the results of multi-population surveys. ${ }^{36}$

Meanwhile, there were brain areas that only appeared frequently in the pain-related abstracts, and not in the mental disorders. The brain areas such as PAG and motor cortex exclusively appeared at a high frequency in the pain-related abstracts. PAG is known to comprise the descending pathway of pain, which exerts influence upon a top-down modulation of pain sensation. It has been demonstrated that the descending pain modulatory circuit can facilitate as well as inhibit pain and that dysfunction of this circuit may lead to the chronification of pain. Although PAG was clustered into a falling group in our study, it showed high levels of relative frequency in general, reflecting the importance of PAG in pain studies. ${ }^{37-42}$

Table 2 Eigenvectors Of The Three Principal Components

\begin{tabular}{|l|l|l|l|l|l|l|l|l|l|l|}
\hline & PFC & Frontal & Parietal & Temporal & Limbic & DIEN & BG & BS & Cerebellum & Corpus \\
\hline PCI & -0.34 & -0.16 & 0.05 & -0.16 & -0.39 & 0.3 & -0.38 & 0.66 & -0.01 & -0.03 \\
PC2 & -0.25 & 0.09 & -0.09 & 0.29 & -0.75 & 0 & 0.48 & -0.2 & -0.03 & 0.03 \\
PC3 & -0.03 & -0.36 & -0.04 & 0.36 & -0.19 & -0.06 & -0.68 & -0.49 & -0.03 & 0 \\
\hline
\end{tabular}

Notes: Each row represents eigenvectors of the corresponding principal component (PC). Each column represents the feature of each brain region group. The value of each cell corresponds to loading indicating how much the feature contributes to the PC.

Abbreviations: Frontal, frontal cortex; Parietal, parietal cortex; Temporal, temporal cortex; Limbic, limbic area; DIEN, diencephalon; BG, basal ganglia; BS, brain stem; Cb, cerebellum; Corpus, corpus callosum. 
Interestingly, the appearance patterns of the cerebellum and motor cortex were notable in pain-related abstracts. Motor cortex, which belongs to the rising cluster, showed a consistent increase in relative frequency from the late 1990s. While most of the regions in the rising cluster frequently co-occurred with other regions to form edges in the subnetwork of hub regions, the motor cortex formed no edges with other areas in the networks in the given threshold. The cerebellum did not show high levels of relative frequency in general; however, it formed edges with hub nodes (ACC and thalamus in stage 3; insula and thalamus in stage 4) in the pain-related brain networks in the last two decades. This tendency reflects the advanced understanding of pain in recent studies regarding the relationship between motor control and pain. ${ }^{43-47}$ The emotional/cognitive brain regions that belonged to the rising cluster or were represented as hubs in the networks have been recognized as important in pain studies in the last two decades. This data suggests that the motor cortex and cerebellum are also likely to play a key role in pain studies in the years to come.

Our results are supposed to be influenced not only by the change in the perspective of the pain mechanism or the researchers' interests, but also by the rapid developments in the brain imaging technologies such as positron emission tomography, computed tomography, and magnetic resonance imaging (MRI). In stage 3 (1996-2005), the configuration of the pain-related brain network changed abruptly from the earlier stages (Figure 7). These changes could partially be attributed to the fact that the portion of the human brain imaging studies in pain research has increased around that time. For instance, increase of cortical regions and decrease of brain stem regions in occurrence pattern could be partially explained by the emergence of functional MRI because it is difficult to obtain data from several complicated regions such as brain stem. ${ }^{48}$

It should be noted that although we describe the brain regions that appeared more than 100 times as pain-related regions, pain-related brain regions are not pain-specific brain regions, ie, pain coding regions. For instance, the insular cortex has been discovered to involve a wide range of functions in humans that encompasses sensory, emotional, and high-level cognition. ${ }^{11}$ And there have been emerging studies showing that ACC is implicated both in cognitive and emotional processes. ${ }^{49}$

One limitation of the study should be noted. Because we only dealt with the abstracts, not the entire manuscripts, the pain-related studies were included for analysis without considering pain types, subjects, and article types. There is a possibility of misinterpretation in the results which may result from this simple criterion. However, the macroscopic changes of pain-related brain regions, which were demonstrated by analyzing a vast number of abstracts, are obviously in line with our current understanding of the pain study trends, supporting the validity of our approach.

In summary, we performed literature-mining analyses in the pain-related abstracts to investigate how the neuroscience field developed over time in terms of its concept on how pain is represented in the brain and compare the research trends of pain with those of mental disorders, and the findings indicate that the regions related to emotional/cognitive aspects of pain have become increasingly important. The relative frequency trajectory of pain-related brain regions has shifted closer to that of mental disorders-related brain regions. Furthermore, we also anticipate that the cerebellum and motor cortex will be actively explored in the pain study because of their notable occurrence patterns. We expect that the literature mining approach used in this study can be applied to other study topics in the future to provide macroscopic insights into the study trends.

\section{Conclusion}

Evolving patterns of the pain-related brain network were examined by analyzing a vast number of abstracts that are impossible to be manually reviewed, and the results are in line with our current understanding of the pain study trends. Temporal changes of pain-related brain regions in the abstracts indicate that emotional/cognitive aspects of pain have been gradually emphasized. The relative frequencies and co-occurrences of brain regions related to the emotional/ cognitive aspects of pain tended to increase consistently. The state-space model showed that the relative frequency trajectory of the pain-related brain regions shifted closer to that of mental disorders-related brain regions over time. Based on the notable occurrence patterns of the cerebellum and motor cortex, these motor-related areas are expected to be actively explored by pain researchers in the future. We expect that the literature mining approach used in this study can be applied to other study topics in the future to provide macroscopic insights into the study trends.

\section{Acknowledgments}

This work was supported by the Gachon University research fund of 2016 (GCU-2016-0493).

\section{Disclosure}

The authors have declared that no competing interests exist in this work. 


\section{References}

1. Melzack R. From the gate to the neuromatrix. Pain. 1999;Suppl 6: S121-126. doi:10.1016/S0304-3959(99)00145-1

2. Loeser JD, Melzack R. Pain: an overview. Lancet. 1999;353 (9164):1607-1609. doi:10.1016/S0140-6736(99)01311-2

3. Apkarian AV, Bushnell MC, Treede RD, Zubieta JK. Human brain mechanisms of pain perception and regulation in health and disease. Eur J Pain. 2005;9(4):463-484. doi:10.1016/j.ejpain.2004.11.001

4. Rainville P. Brain mechanisms of pain affect and pain modulation. Curr Opin Neurobiol. 2002;12(2):195-204. doi:10.1016/s0959-4388 (02)00313-6

5. Price DD. Psychological and neural mechanisms of the affective dimension of pain. Science. 2000;288(5472):1769-1772. doi:10.1126/ science.288.5472.1769

6. Peyron R, Laurent B, Garcia-Larrea L. Functional imaging of brain responses to pain. A review and meta-analysis (2000). Neurophysiol Clin. 2000;30(5):263-288.

7. Wiech K, Ploner M, Tracey I. Neurocognitive aspects of pain perception. Trends Cogn Sci. 2008;12(8):306-313. doi:10.1016/j.tics.2008.05.005

8. Kim C-E, Kim YK, Chung G, et al. Large-scale plastic changes of the brain network in an animal model of neuropathic pain. NeuroImage. 2014;98:203-215. doi:10.1016/j.neuroimage.2014.04.063

9. Apkarian AV. The brain in chronic pain: clinical implications. Pain Manag. 2011;1(6):577-586. doi:10.2217/pmt.11.53

10. Duerden EG, Albanese M-C. Localization of pain-related brain activation: a meta-analysis of neuroimaging data. Hum Brain Mapp. 2013;34(1):109-149. doi:10.1002/hbm.21416

11. Mutschler I, Ball T, Wankerl J, Strigo IA. Pain and emotion in the insular cortex: evidence for functional reorganization in major depression. Neurosci Lett. 2012;520(2):204-209. doi:10.1016/j.neulet.2012.03.095

12. Lopez-Sola M, Pujol J, Hernandez-Ribas R, et al. Effects of duloxetine treatment on brain response to painful stimulation in major depressive disorder. Neuropsychopharmacology. 2010;35(11):23052317. doi:10.1038/npp.2010.108

13. Jensen KB, Kosek E, Wicksell R, et al. Cognitive behavioral therapy increases pain-evoked activation of the prefrontal cortex in patients with fibromyalgia. Pain. 2012;153(7):1495-1503. doi:10.1016/j.pain. 2012.04.010

14. Tegethoff M, Belardi A, Stalujanis E, Meinlschmidt G. Comorbidity of mental disorders and chronic pain: chronology of onset in adolescents of a national representative cohort. J Pain. 2015;16(10):10541064. doi:10.1016/j.jpain.2015.06.009

15. Gerrits MM, van Oppen P, van Marwijk HWJ, Penninx BW, van der Horst HE. Pain and the onset of depressive and anxiety disorders. PAIN®. 2014;155(1):53-59. doi:10.1016/j.pain.2013.09.005

16. Baliki MN, Apkarian AV. Nociception, pain, negative moods, and behavior selection. Neuron. 2015;87(3):474-491. doi:10.1016/j. neuron.2015.06.005

17. Witten IH, Frank E, Hall MA, Pal CJ. Data mining: practical machine learning tools and techniques. Morgan Kaufmann. 2011.

18. Ananiadou S, Kell DB, Tsujii J. Text mining and its potential applications in systems biology. Trends Biotechnol. 2006;24(12):571-579. doi:10.1016/j.tibtech.2006.10.002

19. Chen H, Sharp BM. Content-rich biological network constructed by mining PubMed abstracts. BMC Bioinformatics. 2004;5:147. doi:10.1186/1471-2105-5-147

20. Gonzalez GH, Tahsin T, Goodale BC, Greene AC, Greene CS, Advances R. Emerging applications in text and data mining for biomedical discovery. Brief Bioinform. 2016;17(1):33-42. doi:10.1093/bib/bbv087

21. Rebholz-Schuhmann D, Oellrich A, Hoehndorf R. Text-mining solutions for biomedical research: enabling integrative biology. Nat Rev Genet. 2012;13(12):829-839. doi:10.1038/nrg3337

22. Zhu F, Patumcharoenpol P, Zhang C, et al. Biomedical text mining and its applications in cancer research. $J$ Biomed Inform. 2013;46 (2):200-211. doi:10.1016/j.jbi.2012.10.007
23. Ambert $\mathrm{KH}$, Cohen AM. Text-mining and neuroscience. Int Rev Neurobiol. 2012;103:109-132.

24. French L, Lane S, Xu L, et al. Application and evaluation of automated methods to extract neuroanatomical connectivity statements from free text. Bioinformatics. 2012;28(22):2963-2970. doi:10.1093/ bioinformatics/bts 542

25. Richardet R, Chappelier JC, Telefont M, Hill S. Large-scale extraction of brain connectivity from the neuroscientific literature. Bioinformatics. 2015;31(10):1640-1647. doi:10.1093/bioinformatics/btv025

26. Hearst MA Untangling text data mining. Proceedings of the 37th annual meeting of the Association for Computational Linguistics on Computational Linguistics; 1999; College Park, Maryland.

27. Frijters R, van Vugt M, Smeets R, van Schaik R, de Vlieg J, Alkema W. Literature mining for the discovery of hidden connections between drugs, genes and diseases. PLoS Comput Biol. 2010;6:9. doi:10.1371/journal.pcbi.1000943

28. Shannon P, Markiel A, Ozier O, et al. Cytoscape: a software environment for integrated models of biomolecular interaction networks. Genome Res. 2003;13(11):2498-2504. doi:10.1101/ gr. 1239303

29. Jolliffe IT, Cadima J. Principal component analysis: a review and recent developments. Philos Trans A Math Phys Eng Sci. 2016;374 (2065):20150202. doi:10.1098/rsta.2015.0202

30. Medicine I, Sciences N. Discovering the Brain 4, the Role of the Brain in Mental Illness. Washington, DC: The National Academies Press; 1992.

31. Malfliet A, Coppieters I, Van Wilgen P, et al. Brain changes associated with cognitive and emotional factors in chronic pain: a systematic review. Eur J Pain. 2017;21(5):769-786. doi:10.1002/ejp.1003

32. Thompson JM, Neugebauer V. Cortico-limbic pain mechanisms. Neurosci Lett. 2018.

33. Bair MJ, Robinson RL, Katon W, Kroenke K. Depression and pain comorbidity: a literature review. Arch Intern Med. 2003;163 (20):2433-2445. doi:10.1001/archinte.163.20.2433

34. Gatchel RJ. Comorbidity of chronic pain and mental health disorders: the biopsychosocial perspective. Am Psychol. 2004;59(8):795-805. doi:10.1037/0003-066X.59.8.795

35. Casten RJ, Parmelee PA, Kleban MH, Powell Lawton M, Katz IR. The relationships among anxiety, depression, and pain in a geriatric institutionalized sample. Pain. 1995;61(2):271-276. doi:10.1016/0304-3959(94)00185-h

36. Gureje O, Von Korff M, Kola L, et al. The relation between multiple pains and mental disorders: results from the World Mental Health Surveys. Pain. 2008;135(1-2):82-91. doi:10.1016/ j.pain.2007.05.005

37. Ossipov MH, Dussor GO, Porreca F. Central modulation of pain. $J$ Clin Invest. 2010;120(11):3779-3787. doi:10.1172/JCI43766

38. Fields HL. Pain modulation: expectation, opioid analgesia and virtual pain. Prog Brain Res. 2000;122:245-253. doi:10.1016/s0079-6123(08) 62143-3

39. Lau BK, Vaughan CW. Descending modulation of pain: the GABA disinhibition hypothesis of analgesia. Curr Opin Neurobiol. 2014;29:159-164. doi:10.1016/j.conb.2014.07.010

40. Hemington KS, Coulombe M-A. The periaqueductal gray and descending pain modulation: why should we study them and what role do they play in chronic pain? J Neurophysiol. 2015;114(4):20802083. doi: $10.1152 /$ jn. 00998.2014

41. Zhuo M. Descending facilitation. Mol Pain. 2017;13:1744806917699212. doi:10.1177/1744806917699212

42. Kuner R. Central mechanisms of pathological pain. Nat Med. 2010;16:1258.

43. Cha M, Um SW, Kwon M, Nam TS, Lee BH. Repetitive motor cortex stimulation reinforces the pain modulation circuits of peripheral neuropathic pain. Sci Rep. 2017;7(1):7986. doi:10.1038/s41598-01708208-2 
44. Mercier C, Léonard G. Interactions between pain and the motor cortex: insights from research on phantom limb pain and complex regional pain syndrome. Physiother Can. 2011;63(3):305-314. doi:10.3138/ptc.2010-08p

45. Farina S, Tinazzi M, Le Pera D, Valeriani M. Pain-related modulation of the human motor cortex. Neurol Res. 2003;25(2):130-142. doi:10.1179/016164103101201283

46. Moulton EA, Schmahmann JD, Becerra L, Borsook D. The cerebellum and pain: passive integrator or active participator? Brain Res Rev. 2010;65(1):14-27. doi:10.1016/j.brainresrev.2010.05.005
47. Saab CY, Willis WD. The cerebellum: organization, functions and its role in nociception. Brain Res Rev. 2003;42(1):85-95.

48. Beissner F. Functional MRI of the brain stem: common problems and their solutions. Clin Neuroradiol. 2015;25(2):251-257. doi:10.1007/ s00062-015-0404-0

49. Bush G, Luu P, Posner MI. Cognitive and emotional influences in anterior cingulate cortex. Trends Cogn Sci. 2000;4(6):215-222.

\section{Publish your work in this journal}

The Journal of Pain Research is an international, peer reviewed, open access, online journal that welcomes laboratory and clinical findings in the fields of pain research and the prevention and management of pain. Original research, reviews, symposium reports, hypothesis formation and commentaries are all considered for publication. The manuscript

Submit your manuscript here: https://www.dovepress.com/journal-of-pain-research-journal management system is completely online and includes a very quick and fair peer-review system, which is all easy to use. Visit http:// www.dovepress.com/testimonials.php to read real quotes from published authors. 